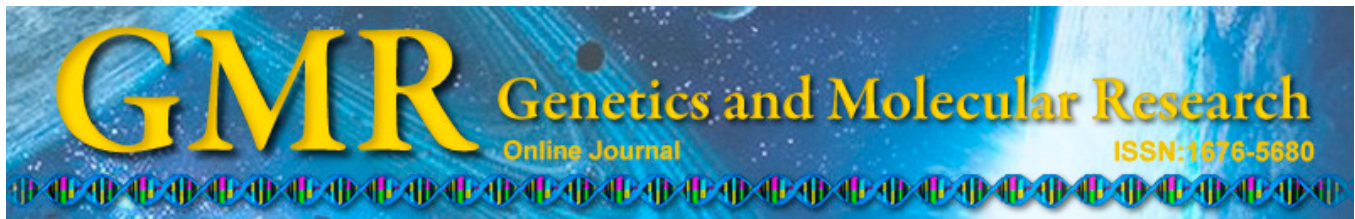

\title{
Manipulation of primer affinity improves high-resolution melting accuracy for imprinted genes
}

\author{
F.V.M. Rubatino, N.V. Carobin, M.L. Freitas, V.T. de Oliveira, \\ R.X. Pietra, P.P.R. Oliveira, A.A. Bosco and F.S. Jehee \\ Instituto de Ensino e Pesquisa da Santa Casa de Belo Horizonte, \\ Belo Horizonte, MG, Brasil \\ Corresponding author: F.V.M. Rubatino \\ E-mail: rubatinofvm@gmail.com
}

Genet. Mol. Res. 14 (3): 7864-7872 (2015)

Received November 12, 2014

Accepted April 6, 2015

Published July 14, 2015

DOI http://dx.doi.org/10.4238/2015.July.14.12

\begin{abstract}
High-resolution melting (HRM) is considered an inexpensive, rapid, and attractive methodology for methylation analysis. In the application of the polymerase chain reaction (PCR) to methylation analysis, amplification efficiencies are biased towards unmethylated, rather than methylated, templates: a phenomenon known as PCR bias. To overcome PCR bias, primers that include $\mathrm{CpG}$ site(s) and are fully complementary to the methylated sequence have been proposed. However, genes mapped within imprinted regions usually present higher methylation levels, and an unusual PCR bias towards the methylated template can therefore arise. The manipulation of primer affinity attempts to overcome this problem. We attempted to show that mismatches at the primer's methylated binding sites increase the area between the 50 and $100 \%$ methylation plots on the melting curves, and may increase HRM accuracy for samples that have high methylation levels. Sets of primers for imprinted genes that included $\mathrm{CpG}$ sites at their binding sequences were designed, and were complementary to methylated or unmethylated templates. Primers fully complementary
\end{abstract}


to methylated templates produced a very small area between the 50 and $100 \%$ methylation plots. When using primers that were fully complementary to the unmethylated sequence, we were able to increase the area between the 50 and $100 \%$ methylation plots. Therefore, when samples are highly methylated, such as targets in genes mapped in imprinted regions, we propose that primers should favor amplification of the rarest, unmethylated sequence. Primers may be designed to include one $\mathrm{CpG}$ at its binding site and be fully complementary to the unmethylated template.

Key words: Imprinted genes; Methylation; High-resolution melting; Epigenetics; Polymerase chain reaction

\section{INTRODUCTION}

Cytosine methylation at promoter regions is an important mechanism of gene expression regulation and has been investigated extensively. The process may be related to three important events: 1) germinative imprinting, when genes or gene clusters are marked during gametogenesis depending on their parental origin, resulting in a mono-allelic expression in a diploid individual; 2) somatic imprinting, when parental marking concerns time and specific tissues; and 3) epigenetic changes associated with physiological gene expression requirements or some diseases such as cancer (Prickett and Oakey, 2012).

Among the methodologies for methylation pattern access, of note are methylationspecific polymerase chain reaction (PCR), bisulfite sequencing, and methylation-sensitive melting curves analysis (MS-MCA) (Hernández et al., 2013). MS-MCA is an attractive approach to quantification analyses because it is inexpensive and allows several samples to be analyzed simultaneously (Worm et al., 2001; Wojdacz and Hansen, 2006). These approaches use DNA after bisulfite treatment and require a combination of a PCR thermocycler and a fluorescence detector. The assays require an intercalating fluorescent dye that is able to emit a fluorescence signal when oligonucleotide double strands are present (Life Technologies Corporation, 2010). During bisulfite treatment, unmethylated cytosines are converted to uracil and are substituted by thymine in PCR; bisulfite does not convert methylated cytosines. This induces DNA sequence differences that depend on the initial methylation status. Therefore, methylated sequences have greater numbers of $\mathrm{C}$ and $\mathrm{G}$ than unmethylated sequences, producing a difference in the melting curve profiles (Worm et al., 2001; Tost, 2009; Hernández et al., 2013). Owing to the evolution of intercalating dye and real-time PCR platforms, melting curve analyses have been termed high resolution melting (HRM) (Tost, 2009).

MS-MCA PCR uses a single pair of primers that is able to amplify both methylated and unmethylated sequences indiscriminately (Guldberg et al., 2002). This approach poses two main problems: the difficulty of designing primers at promoter regions without including CpG sites; and PCR amplification efficiency is not usually the same for methylated and unmethylated templates. PCR amplification of unmethylated templates is often more efficient than of methylated templates, which is PCR bias (Wojdacz and Hansen, 2006).

Wojdacz and Dobrovic (2007) proposed a new approach to studying epigenetic changes in cancer using methylation-sensitive high resolution melting (MS-HRM). This approach is particularly innovative in its primer design and its ability to reverse PCR bias (Wojdacz et al., 2008). 
The primer design proposed by Wojdacz and Dobrovic (2007) introduces new challenges for the determination of the methylation status of imprinted genes. In some genomic regions or in specific tissues, high methylation levels are expected. Although unmethylated sequences usually have a better PCR amplification efficiency, they are much rarer than methylated ones. This fact may create an unusual PCR bias towards the methylated sequence. Our study aims to overcome the unusual bias towards highly methylated samples present in PCR amplification by manipulating the primer affinity.

\section{MATERIAL AND METHODS}

\section{Samples and ethics statement}

Peripheral blood was collected in ethylenediaminetetraacetic acid tubes from 10 heathy adult subjects who signed written informed consent. This project was approved by the Santa Casa Hospital Human Research Ethics Committee.

\section{DNA extraction and bisulfite treatment}

DNA was extracted using a Gentra Puregene Blood Kit from Qiagen, Maryland, USA, following the manufacturer instructions. Bisulfite treatment was performed using an EpiTect Plus DNA Bisulfite Kit from Qiagen, Hilden, German, following the manufacturer instructions.

\section{Primers design and sequences}

We designed primers for MAGEL2 (NG_016776.1), SNRPN (NG_012958.1), and NECDIN (NG_009380.1), which have exclusively paternal expression and are mapped within the imprinted Prader-Willi/Angelman syndrome region, 15q11.2-q13 (Cassidy et al., 2012). Primers were designed using the Methyl Primer Express v1.0 and the MethPrimer software ( $\mathrm{Li}$ and Dahiya 2002), and included $\mathrm{CpG}$ sites as close to the $5^{\prime}$ end as possible. The first set of primers, MAGEL2MU, were designed according to Wojdacz et al. (2008). MAGEL2MU establishes a perfect match to the methylated template and has one mismatch to the unmethylated template at both the forward and reverse sequences. The second set of primers, MAGEL2MM, was designed with a perfect match to the unmethylated template and has a single mismatch at the forward sequence to the methylated binding sequence. NECDINMM and SNRPNMM primers are fully complementary to the unmethylated template and NECDINMM has one mismatch in the forward and reverse sequences; the SNRPNMM set has one mismatch in the reverse sequence at the methylated template. These two primers (NECDINMM and SNRPNMM) were designed to test the reproducibility of the new approach for other targets. Table 1 shows the primer sequences and their PCR product sizes. CpG sites are highlighted in red. Figure 1 shows MAGEL2MU and MAGEL2MM primers at their binding sites.

\section{PCR conditions and controls}

Primer concentrations were as follows: $0.125 \mu \mathrm{M}$ for each MAGEL2MU primer and $0.175 \mu \mathrm{M}$ for each MAGEL2MM, NECDINMM, or SNRPNMM primer. Reactions with $M A G E L 2$ and NECDIN genes were conducted with the commercially available bisulfite-con- 
verted methylated and unmethylated control DNA (EpiTect PCR control DNA set, Qiagen). Bisulfite-converted DNA from Prader-Willi (100\% methylated) and Angelman ( $0 \%$ methylated) subjects were used as DNA controls for $S N R P N$ gene reactions. All PCR reactions were carried out in a final volume of $10 \mu \mathrm{L}$ containing 2 ng DNA, 1X MeltDoctor Master Mix from Applied Biosystems, and an additional $2 \mathrm{mM} \mathrm{MgCl}_{2}$. All reactions were performed in duplicate using the ABI 7500 Fast System. The PCR programs are given in Table 2.

\begin{tabular}{|c|c|c|c|}
\hline Primer & Forward sequence (5' to $3^{\prime}$ ) & Reverse sequence (5' to 3') & Product size (bp) \\
\hline MAGEL2MU & GTTTTTTCGTAGGTTTTTTAGGGTT & TTACTACGTAACCTACCATCCTAA & 102 \\
\hline MAGEL2MM & GTTTTTTTGTAGGTTTTTTAGGGTT & AAATAACCTACCAACCTTACT & 118 \\
\hline SNRPNMM & GGAGTTGGGATTTTTGTATT & TAACCACTCCTCAAACAAATA & 171 \\
\hline NECDINMM & GATGTAGAGGTTTTGTTTTTGTT & CAAAACCCCAAAACTACTATACA & 123 \\
\hline
\end{tabular}

(1) AAGGGCTCCTCCGCAGGTTCCCCAGGGCCCGCAGGCACCCCCAGCGCAGCTAGCCACACC

||||$|:|::|::+:||||||::::|||||::++:|||:| 1:::::||++:|||:|||::|:|::$ :

(2) AAGGGTTTTTTCGTAGGTTTTTTAGGGTTCGTAGGTATTTTTAGCGTAGTTAGTTATATT

A 5' GTTTtTtCGtagGtTttTtaggGtT 3'

B 5' GTTTtTTTGTAGGTtTTTAGGGTT 3'

(contimuation)

(1) CCCGGGCTGGCAGGCGACCTCGCCAGGATGGCAGGCCACGCAGCAAGGCTGGCAGGCCACTC $::++||:|||:|||++|::|++::||||||||:||||::|++:|||:||||:||||:|||::||:|:$

(2) TTCGGGTTGGTAGGCGATTTCGTTAGGATGGTAGGTTACGTAGTAAGGTTGGTAGGTTATTT

C 3' AATCCTACCATCCAATGCATCATT 5'

D 3' 'TCATTCCAACCATCCAATAAA 5'

Figure 1. MAGEL2 promoter sequences adapted from the MethPrimer software. Line one represents the original DNA sequence; line two shows the DNA sequence after bisulfite treatment. "++" refers to CpG sites and ":" refers to $\mathrm{C}$ modified to T after bisulfite treatment. (A) MAGEL2MU forward sequence; (B) MAGEL2MM forward sequence; (C) MAGEL2MU reverse sequence; and (D) MAGEL2MM reverse sequence.

Table 2. Thermocycler programs.
\begin{tabular}{lcll}
\hline Primer & Cycles & Denaturation & Annealing/Extension \\
\hline MAGEL2MM & 45 & $95^{\circ} \mathrm{C} / 10 \mathrm{~min}$ & $61^{\circ}, 62^{\circ}, 63^{\circ}$ or $64^{\circ} \mathrm{C} / 60 \mathrm{~s}$ \\
MAGEL2MU & 45 & $95^{\circ} \mathrm{C} / 10 \mathrm{~min}$ & $58.5^{\circ}$ or $60^{\circ} \mathrm{C} / 60 \mathrm{~s}$ \\
SNRPNMM & 45 & $95^{\circ} \mathrm{C} / 10 \mathrm{~min}$ & $52.5^{\circ} / 20 \mathrm{~s}, 67^{\circ} \mathrm{C} / 40 \mathrm{~s}$ \\
NECDINMM & 45 & $95^{\circ} \mathrm{C} / 10 \mathrm{~min}$ & $64^{\circ} \mathrm{C} / 60 \mathrm{~s}$ \\
\hline
\end{tabular}

\section{Determination of the standard curve and methylation samples}

The standard curve was made from samples with known different levels of methylation $(0,50,75,90,99$, and 100\%). PCR amplifications using MAGEL2MM were carried out at $63^{\circ} \mathrm{C}$ both for annealing and extension, after other tests. Data for the difference of fluorescence 
plot, using a $0 \%$ methylated sample as a baseline, were plotted on the y-axis and the known values of methylation were plotted on the x-axis. To determinate methylation samples from subjects, mean values of the difference of fluorescence plot were applied to the equation of the standard curve.

\section{RESULTS}

For the PCR primer MAGEL2MU at $60^{\circ} \mathrm{C}$, both for annealing and extension, the methylated sequence amplification was much higher than in the unmethylated sequence, as reflected by the small area between the plots for 50 and $100 \%$ methylation samples. At $58.5^{\circ} \mathrm{C}$, the area between the 50 and $100 \%$ plots of the methylated samples increased slightly (Figure $2 \mathrm{~A}$ and $\mathrm{B})$. Annealing temperatures lower than $58.5^{\circ} \mathrm{C}$ gave rise to unspecific PCR products without improving the area between the 50 and $100 \%$ methylation plot lines. Further changes in primers and $\mathrm{MgCl}_{2}$ concentration had no useful effect (data not shown).
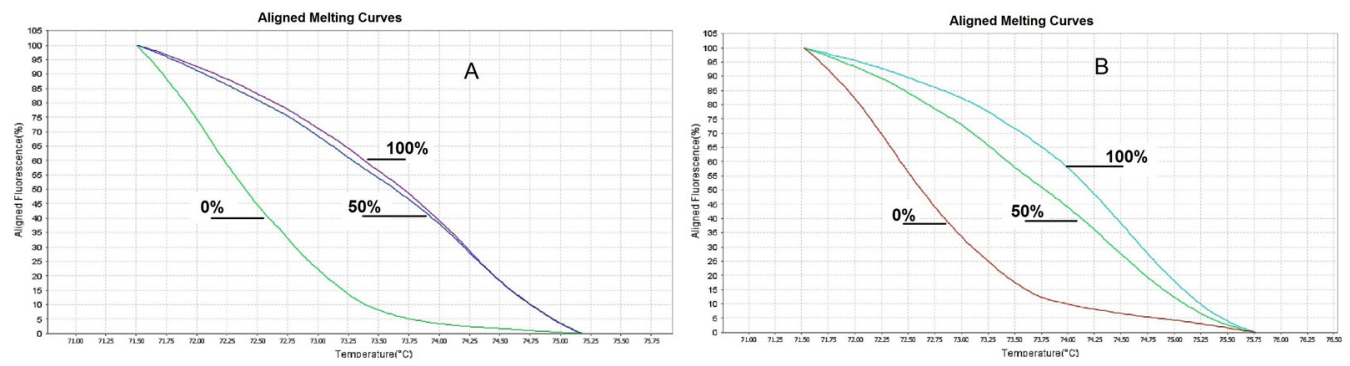

Figure 2. Aligned melting curves using MAGEL2MU primers. A. Aligned melting curve for polymerase chain reaction $(\mathrm{PCR})$ at $60^{\circ} \mathrm{C}$, both for annealing and extension. Zero percent methylated is shown in green, $50 \%$ in blue, and $100 \%$ in purple. B. Aligned melting curves for PCR at $58.5^{\circ} \mathrm{C}$ both for annealing and extension. Zero percent methylated is shown in red, $50 \%$ in green, and $100 \%$ in blue.

With the objective of selecting the best PCR conditions and the best ratio of methylated and unmethylated template amplification, reactions using primer MAGEL2MM with controls were carried out at $61^{\circ}, 62^{\circ}, 63^{\circ}$, and $64^{\circ} \mathrm{C}$ both for annealing and extension. By manipulation of the annealing and extension temperatures, the areas between the 0,50 , and $100 \%$ methylation plots were adjusted. At temperatures higher than $62^{\circ} \mathrm{C}$, the aligned melting curves displayed a plot area between the 50 and $100 \%$ methylation melting curves larger than that between the 0 and $50 \%$ plots, as shown in Figure 3.

The methylation status of MAGEL2 for the 10 subjects was determined with annealing and extension at $63^{\circ} \mathrm{C}$. The reaction was accompanied by a standard curve that showed $\mathrm{R}^{2}=$ 0.9806 , which proved that the results were reliable (Figure 4). Using these reaction conditions it was possible to accurately analyze samples from all subjects that had methylation levels above $90 \%$ (results not shown).

In an attempt to test the reproducibility of these conditions for other targets, PCRs with primers NECDINMM and SNRPNMM were carried out. For all reactions, we were able to produce a larger area between the 50 and $100 \%$ methylation plots than between the 0 and $50 \%$ plots on the aligned melting curves (Figure 5). As with MAGEL2, the methylation statuses of NECDIN and $S N R P N$ for the 10 subjects were determined (data not shown). 

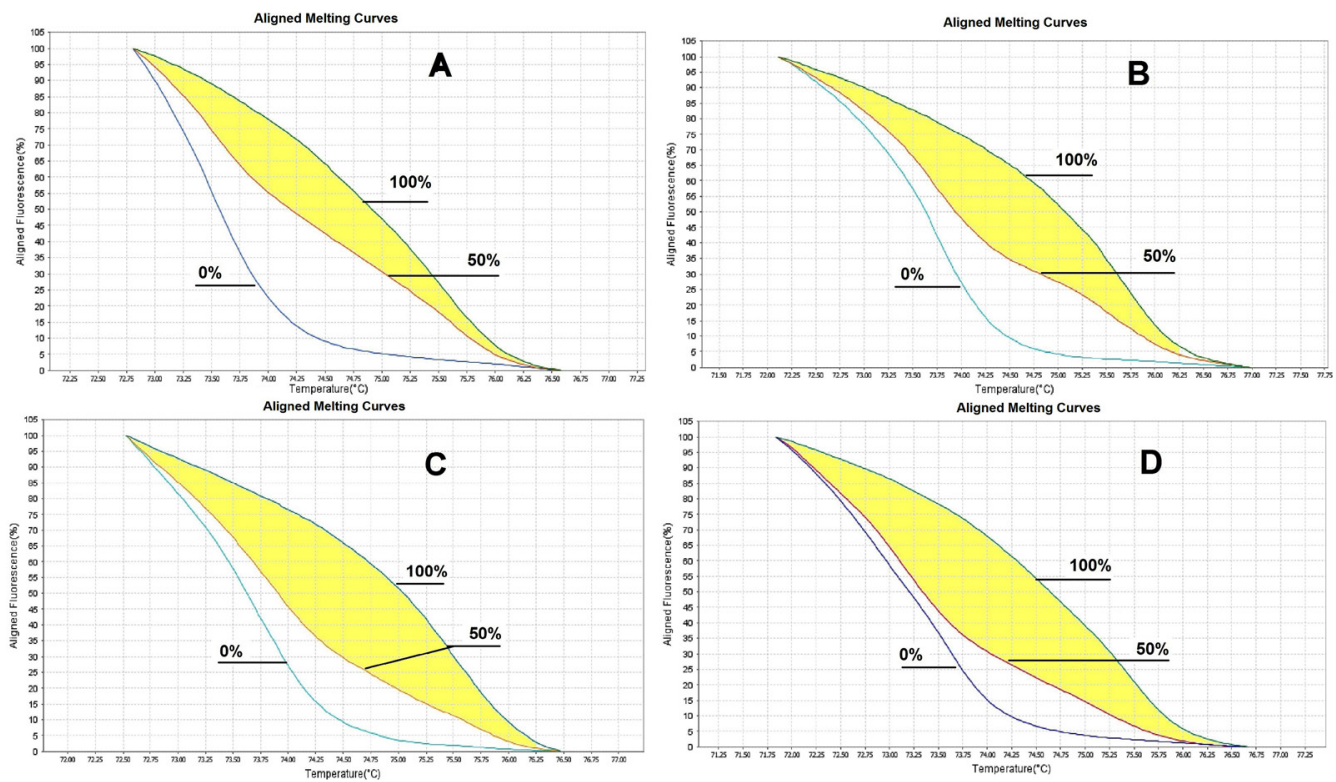

Figure 3. Aligned melting curves for polymerase chain reaction (PCR) using MAGEL2MM primers, both for annealing and extension, at $61^{\circ} \mathrm{C}(\mathbf{A}), 62^{\circ} \mathrm{C}(\mathbf{B}), 63^{\circ} \mathrm{C}(\mathbf{C})$, and $64^{\circ} \mathrm{C}(\mathbf{D})$. Melting curves for 100,50 , and $0 \%$ methylation are shown in green, red, and blue, respectively. The area between the 50 and $100 \%$ methylation plots is shown in yellow.

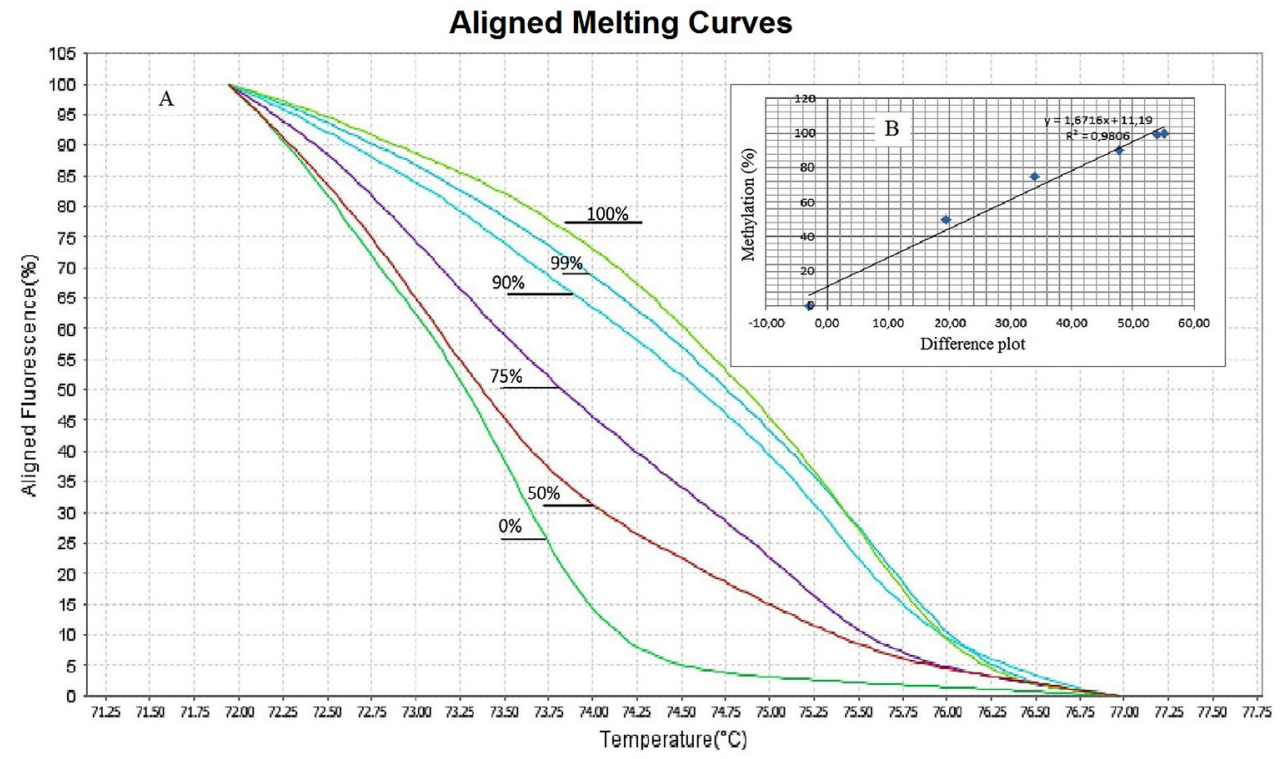

Figure 4. Aligned melting curves for polymerase chain reaction (PCR) using MAGEL2MM primers. (A) From left to right, aligned melting curves for 0, 50, 75, 90, 99, and 100\% methylated samples. (B) Standard curve. 

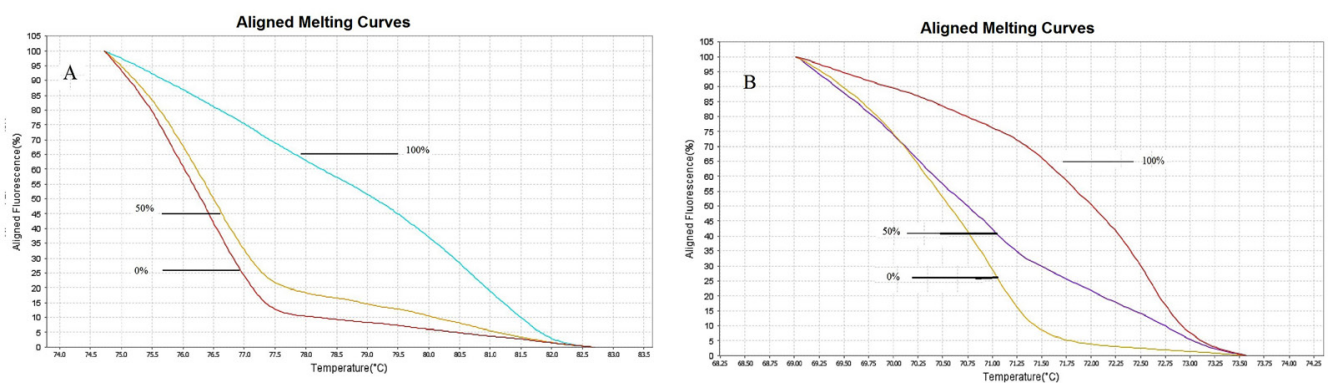

Figure 5. Aligned melting curves for polymerase chain reaction (PCR) with controls: 0 , 50, and 100\% methylation. SNRPNMM primers (A) and NECDINMM primers (B). A larger area between the 50 and $100 \%$ methylation plots than between the 0 and $50 \%$ methylation plots can be seen for both primers.

\section{DISCUSSION}

In methylation studies, amplification efficiency is largely dependent on certain PCR variables: extension temperature, amplicon size, amplicon $\mathrm{C}$ and $\mathrm{G}$ content, and primer affinity to its binding site. Unmethylated sequences are usually amplified more than methylated sequences, particularly at lower temperatures; smaller amplicons and sequences with fewer $\mathrm{CpG}$ sites have higher amplification efficiencies than longer amplicons containing more $\mathrm{CpG}$ sites (Wojdacz and Hansen, 2006; Hernández et al., 2013). Primer affinity to its binding site may be manipulated by design and annealing temperature adjustments (Wojdacz and Hansen, 2006; Wojdacz et al., 2008, 2009).

Wojdacz et al. manipulated primer affinity to improve methylated template PCR amplification (Wojdacz and Hansen, 2006; Wojdacz and Dobrovic, 2007). The authors designed primers that were fully complementary to the methylated binding template, making one or two mismatches at $\mathrm{CpG}$ sites within the unmethylated binding sequence (Wojdacz and Hansen, 2006; Wojdacz and Dobrovic, 2007; Wojdacz et al., 2009). In their assay, the area under the $50 \%$ methylation plot was larger than that above, resulting in a large area in which low methylation fractions are dispersed. This results in high accuracy, particularly for low methylation fraction samples, and has proved very pertinent to cancer studies because cancer samples usually have low methylation levels (Wojdacz and Hansen, 2006; Wojdacz and Dobrovic, 2007; Wojdacz et al., 2008, 2009, 2011; Mencalha et al., 2013).

In our assay, for MAGEL2MM and MAGEL2MU, we worked with small amplicons of similar sequences, so the main feature that influenced PCR amplification was primer affinity. The MAGEL2MU primers resulted in a small area between the 50 and $100 \%$ methylation melting curve plots. In an attempt to increase unmethylated sequence amplification, both annealing and extension temperatures were decreased to $58.5^{\circ} \mathrm{C}$, as suggested by Wojdacz et al. (Wojdacz and Hansen, 2006; Wojdacz and Dobrovic, 2007). A slight change in amplification occurred and was reflected in the small change in the area between the 50 and $100 \%$ methylation melting curve plots. However, the area above the 50\% methylation plot remained smaller than the area under it. Ideally, the samples should be dispersed in an area that is large enough to detect small differences among them.

The MAGEL2MM primers allowed for better amplification of unmethylated templates at all tested temperatures and they enabled us to work at higher temperatures, decreas- 
ing further primer dimer and unspecific product formation. As intended, our results showed that improving unmethylated template amplification increased the area between the 50 and $100 \%$ methylation melting curve plots (Figure 6). The high levels of methylation found in MAGEL2 in the subjects analyzed were consistent with the low expression levels of MAGEL2 present in white blood cells (Lee et al., 2000). We tested the same approach for other targets, $N E C D I N$ and $S N R P N$ promoter regions, which were also expected to present high methylation levels. The ability to produce a larger area between the 50 and $100 \%$ methylation melting curve plots using primers with mismatches to the methylated template was reproducible in all tested targets.

The effects of both annealing and extension temperature changes may change the ratio of methylated and unmethylated amplifications and their melting curves. The effects of temperature changes may be different among reactions because using both lower annealing and extension temperatures would favor unspecific annealing, mostly at methylated templates, increasing their amplification ratio. However, higher annealing and extension temperatures might favor unmethylated annealing but would improve methylated amplification. To find the perfect amplification conditions it is essential to test a wide range and combination of annealing and extension temperatures for each target.

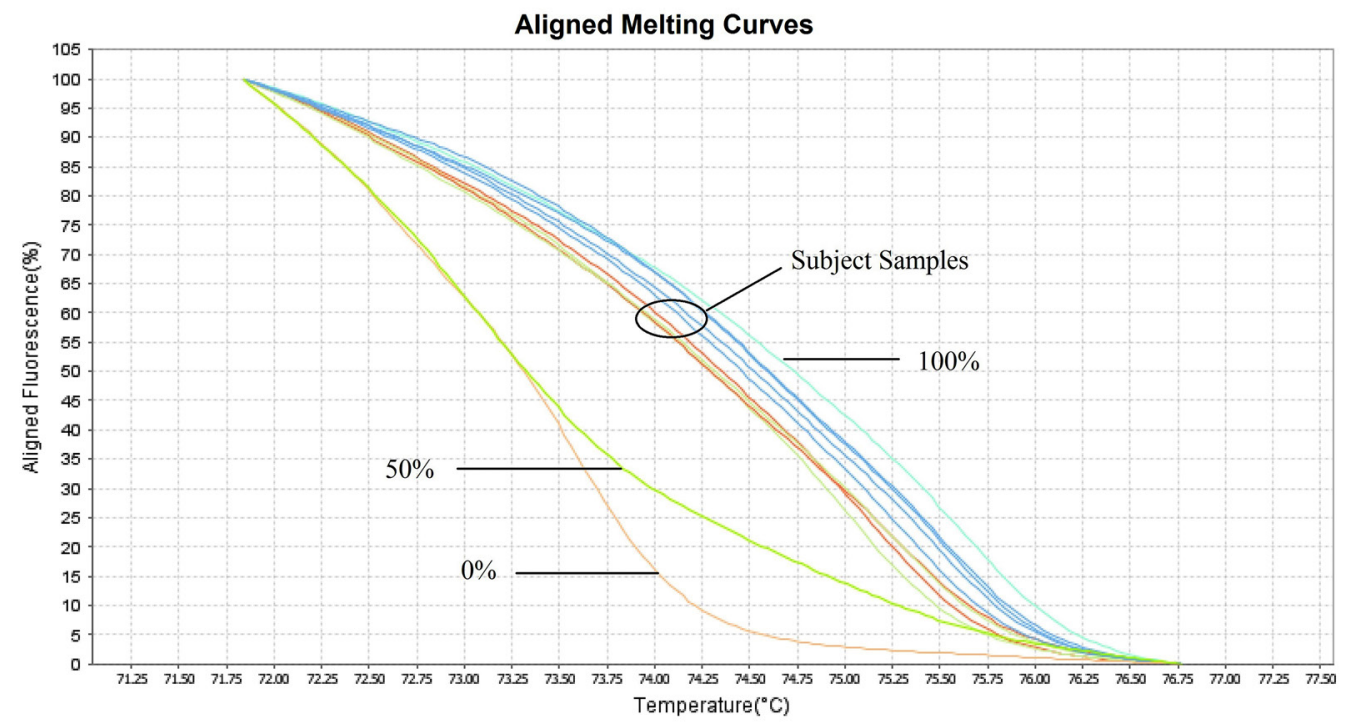

Figure 6. Aligned melting curves for polymerase chain reaction (PCR) with MAGEL2MM primers at $63^{\circ} \mathrm{C}$. The subject samples show small differences at high methylation levels.

The MS-HRM assay supports a potential flexibility for methylation fraction ranges. By controlling and balancing PCR amplification efficiency between methylated and unmethylated sequences, it is possible to adjust MS-HRM assay sensitivity for different methylation level analyses. By redirecting PCR amplification, we may change the melting curves in MSHRM, which change the areas between the 0 and $50 \%$ and the 50 and $100 \%$ methylation melting curve plots. However, to increase the area between the 50 and $100 \%$ methylation plots, where highly methylated samples are plotted, PCR must favor the unmethylated amplification 
sequence over the methylated one. Therefore, in MS-HRM, prior to the assay design it is essential to know the supposed level of methylation of the sample, which may be of great value when designing primer sets. Depending on the methylation profile of the samples studied, primers should be designed with mismatches to the methylated or the unmethylated template, thereby guaranteeing an adequate amplification of the rarest target. Therefore we suggest that when studying genes mapped within imprinted regions and/or high methylation level targets, the reaction must favor the unmethylated allele, which should be accomplished by primer affinity manipulation.

\section{Conflicts of interest}

The authors declare no conflict of interest.

\section{ACKNOWLEDGMENTS}

The authors would like to thank the following funding agencies: the Fundação de Amparo à Pesquisa de Minas Gerais/FAPEMIG; the Coordenação de Aperfeiçoamento de Pessoal de Nível Superior/CAPES; the Conselho Nacional de Desenvolvimento Científico e Tecnológico/CNPq; and the Instituto de Ensino e Pesquisa da Santa Casa de Belo Horizonte/ IEP-SCBH.

\section{REFERENCES}

Cassidy SB, Schwartz S, Miller JL and Driscoll DJ (2012). Prader-Willi syndrome. Genet. Med. 14: 10-26.

Guldberg P, Worm J and Grønbaek K (2002). Profiling DNA methylation by melting analysis. Methods 27: 121-127.

Hernández HG, Tse MY, Pang SC, Arboleda H, et al. (2013). Optimizing methodologies for PCR-based DNA methylation analysis. Biotechniques 55: 181-197.

Lee S, Kozlov S, Hernandez L, Chamberlain SJ, et al. (2000). Expression and imprinting of MAGEL2 suggest a role in Prader-Willi syndrome and the homologous murine imprinting phenotype. Hum. Mol. Genet. 9: 1813-1819.

Li LC and Dahiya R (2002). MethPrimer: designing primers for methylation PCRs. Bioinformatics 18: 1427-1431.

Life Technologies Corporation (2010). A Guide to High Resolution Melting (HRM) Analysis. Available at [https:// sbmsaiumu.files.wordpress.com/2013/06/hrm-guide.pdf]. Accessed February 14, 2015.

Mencalha AL, Rodrigues EF, Abdelhay E, Fernandez TS (2013). Accurate monitoring of promoter gene methylation with high-resolution melting polymerase chain reaction using the ABCB1 gene as a model. Genet. Mol. Res. 12: 714-722.

Prickett AR and Oakey RJ (2012). A survey of tissue-specific genomic imprinting in mammals. Mol. Genet. Genomics 287: 621-630.

Tost J (2009). DNA Methylation: Methods and Protocols. 2nd edn. Humana Press, New York.

Wojdacz TK and Hansen LL (2006). Reversal of PCR bias for improved sensitivity of the DNA methylation melting curve assay. Biotechniques 41: 274-278.

Wojdacz TK and Dobrovic A (2007). Methylation-sensitive high resolution melting (MS-HRM): a new approach for sensitive and high-throughput assessment of methylation. Nucleic Acids Res. 35: e41.

Wojdacz TK, Hansen LL and Dobrovic A (2008). A new approach to primer design for the control of PCR bias in methylation studies. BMC Res. Notes 1: 54 .

Wojdacz TK, Borgbo T and Hansen LL (2009). Primer design versus PCR bias in methylation independent PCR amplifications. Epigenetics 4: 231-234.

Wojdacz TK, Thestrup BB, Overgaard J and Hansen LL (2011). Methylation of cancer related genes in tumor and peripheral blood DNA from the same breast cancer patient as two independent events. Diagn. Pathol. 6: 116.

Worm J, Aggerholm A and Guldberg P (2001). In-tube DNA methylation profiling by fluorescence melting curve analysis. Clin. Chem. 47: 1183-1189. 